\title{
LOCAL UNIQUENESS IN BOUNDARY PROBLEMS $†$
}

\author{
by M. H. MARTIN \\ (Received 7th December 1968)
}

\section{Introduction}

The study of periodic, irrotational waves of finite amplitude in an incompressible fluid of infinite depth was reduced by Levi-Civita (1) to the determination of a function

$$
\omega(\zeta)=\theta+i \tau, \quad \omega(0)=0, \quad \zeta=\rho e^{i \sigma},
$$

regular analytic in the interior of the unit circle $\rho=1$ and which satisfies the condition

$$
\frac{d \tau}{d \sigma}=p e^{-3 \tau} \sin \theta, \quad p=\text { const. }
$$

on the boundary. Here $\theta$ is the angle of inclination of the flow, $q=c e^{\tau}$ is its speed and $c$ is the velocity of wave propagation.

The uniqueness of the solution to the problem is a question of long standing, raised by Levi-Civita himself. Both Levi-Civita and Lichtenstein (2) demonstrated existence and uniqueness if $p$ is sufficiently close to a positive integer. Levi-Civita used Cauchy's method of "Calcul des Limites" and Lichtenstein the method of non-linear integral equations. In 1957 Stoker (3) returned to the question using the methods of modern functional analysis; in particular an iteration procedure devised by Littman and Nirenberg. Recently Dunninger and the author (4), using elementary methods, obtained the following result on uniqueness.

Given a solution $\omega_{1}(\zeta)=\theta_{1}+i \tau_{1}$, no other solution $\omega_{2}(\zeta)=\theta_{2}+i \tau_{2}$ exists for which

$$
\begin{aligned}
& \left(\frac{\sin \frac{1}{2} \theta_{2}}{\sin \frac{1}{2} \theta_{1}}\right)^{\frac{4}{3}}<\frac{q_{2}}{q_{1}}<\left(\frac{\cos \frac{1}{2} \theta_{2}}{\cos \frac{1}{2} \theta_{1}}\right)^{\frac{4}{3}} \text { if }\left|\theta_{2}\right|<\left|\theta_{1}\right|, \\
& \left(\frac{\cos \frac{1}{2} \theta_{2}}{\cos \frac{1}{2} \theta_{1}}\right)^{\frac{4}{4}}<\frac{q_{2}}{q_{1}}<\left(\frac{\sin \frac{1}{2} \theta_{2}}{\sin \frac{1}{2} \theta_{1}}\right)^{\frac{4}{3}} \text { if }\left|\theta_{2}\right|>\left|\theta_{1}\right|,
\end{aligned}
$$

provided $\lambda^{-1}, \lambda=\sin \theta_{2} / \sin \theta_{1}$ are regular and $\left|\theta_{1}\right|+\left|\theta_{2}\right|<\pi$ in $\rho \leqq 1$.

The inequalities (1.1) are portrayed in the hodograph plane (the plane with polar coordinates $q, \theta)$ by the shaded areas in Fig. 1a. Under the conditions stated no second solution exists for which the hodograph point $\left(q_{2}, \theta_{2}\right)$ lies in

$\dagger$ Research supported under contract DA-HC04-(AROD) 67-C-0062 of the Army Research Office, Durham, and the University of Maryland. 
the shaded region attached to the hodograph point $\left(q_{1}, \theta_{1}\right)$, both points $\left(q_{1}, \theta_{1}\right)$, $\left(q_{2}, \theta_{2}\right)$ corresponding to the same point $\zeta$ in the unit circle $\rho<1$. One would prefer a uniqueness theorem without the inequalities (1.1), i.e., one in which the shaded region attached to $\left(q_{1}, \theta_{1}\right)$ is the entire plane, or, if this is not possible, a full neighbourhood of $\left(q_{1}, \theta_{1}\right)$; for example, the circular region in Fig. $1 \mathrm{~b}$. One arrives in this way at a concept of local uniqueness to which we shall return in a moment.

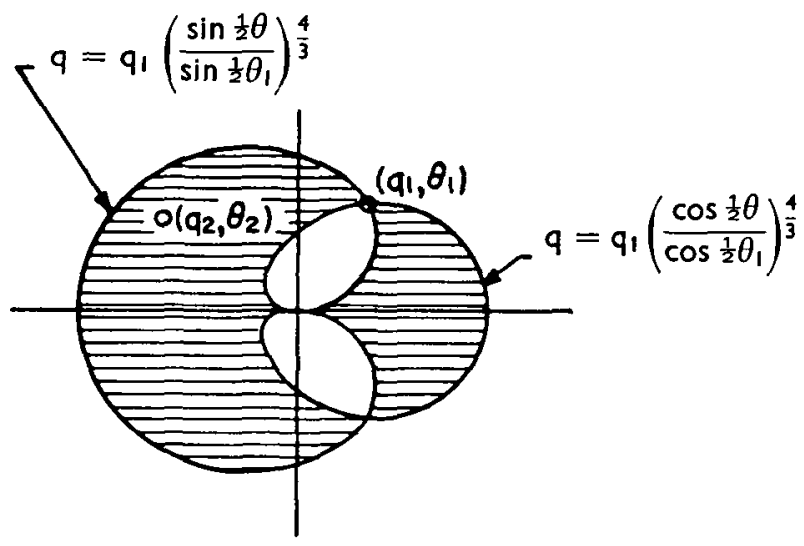

FIG. 1a

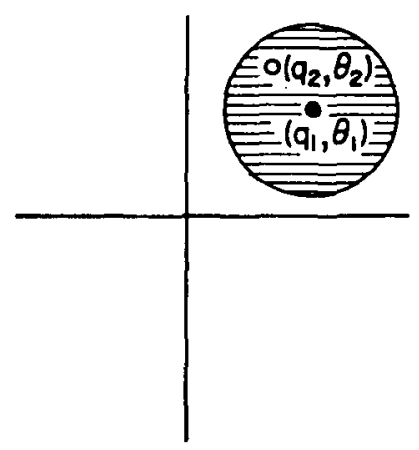

FIG. 1b

The general problem treated in the paper which includes the Levi-Civita problem is the following.

Determine a function $w=u+i v$ of the complex variable $z=x+i y$ regular analytic in a region $S$ of the z-plane bounded by an analytic curve $C$ upon which

$$
u_{n}=h(s) f(u, v)
$$

where $u_{n}$ denotes the external normal derivative of $u$ on $C$, and $h, f$ are given analytic functions of their arguments, with $s$ denoting some parameter on $C$, e.g., the arc length.

Prompted by the above results on the Levi-Civita problem, we seek those functions $f(u, v)$ in (1.2) for which a solution $w$ is locally $\rho$-unique in the following sense, as defined by Cushing (5).

A solution $w_{1}=u_{1}+i v_{1}$ of (1.2) is locally $\rho$-unique if a non-negative function $\dagger \rho=\rho\left(u_{1}, v_{1}\right)$ can be found such that there is no second solution

for which

$$
w_{2}=u_{2}+i v_{2}
$$

$$
0 \leqq\left|w_{1}(z)-w_{2}(z)\right| \leqq \rho, \quad z \in S .
$$

$\dagger$ Cushing (5) takes $\rho=\rho(x, y)$, but the choice $\rho=\rho\left(u_{1}, v_{1}\right)$ is more convenient for our purposes. 
Obviously if the solution to (1.2) is unique in the ordinary sense, it is locally $\rho$-unique, but the converse need not be true.

To avoid difficulties on the boundary $C$ we assume that any solution $w$ is regular analytic in a region $R \supset S+C$.

\section{Preliminary results}

In this section we collect a number of results for later use.

The integral identity

$$
\int_{C} \tau\left(f_{2} \frac{\partial u_{1}}{\partial n}-f_{1} \frac{\partial u_{2}}{\partial n}\right) d s=\int_{S} Q d S,
$$

a ready consequence of Gauss' theorem, is basic to our considerations. Here $\tau=\tau\left(u_{1}, u_{2}, u_{3}, u_{4}\right)$ is an arbitrary function of the real and imaginary parts of two analytic functions

and

$$
w_{1}=u_{1}+i u_{3}, \quad w_{2}=u_{2}+i u_{4},
$$

$$
f_{1}=f_{1}\left(u_{1}, u_{3}\right), f_{2}=f_{2}\left(u_{2}, u_{4}\right),
$$

are assumed given in advance. $Q$ is a quadratic form

$$
\begin{aligned}
& Q=a p_{1}^{2}+2 b p_{1} p_{2}+c p_{2}^{2}+2 d\left(p_{2} p_{3}-p_{1} p_{4}\right)+a p_{3}^{2}+2 b p_{3} p_{4}+c p_{4}^{2}, \\
& p_{k}=\frac{\partial u_{k}}{\partial x},
\end{aligned}
$$

in the partial derivatives $p_{k}$ with coefficients

$$
\begin{array}{ll}
a=f_{2} \tau_{u_{1}}, & 2 b=\left(f_{2} \tau\right)_{u_{2}}-\left(f_{1} \tau\right)_{u_{1}}, \\
c=-f_{1} \tau_{u_{2}}, & 2 d=-\left(f_{1} \tau\right)_{u_{3}}-\left(f_{2} \tau\right)_{u_{4}},
\end{array}
$$

which are determined, once the function $\tau$ has been selected.

If $w_{1}, w_{2}$ are two solutions to (1.2), i.e., if

$$
\frac{\partial u_{1}}{\partial n}=h(s) f\left(u_{1}, u_{3}\right), \quad \frac{\partial u_{2}}{\partial n}=h(s) f\left(u_{2}, u_{4}\right)
$$

hold on $C$ and if we take

$$
f_{1}=f\left(u_{1}, u_{3}\right), \quad f_{2}=f\left(u_{2}, u_{4}\right),
$$

the identity (2.1) implies $Q \equiv 0$ in $S$, provided $Q$ is definite, or at least semidefinite. If $Q$ is definite (semi-definite) there is no loss in generality in assuming $Q$ positive definite (positive semi-definite), since $Q$ changes its sign if $\tau$ is replaced by $-\tau$.

If $E_{4}$ denotes the four dimensional space of the variables $u_{1}, u_{2}, u_{3}, u_{4}$, the domain $D$ in which $Q$ is positive definite obviously depends on the function $\tau$ and one seeks a function $\tau$ that will make $D$ as large as possible in view of the following lemma (4). 
Lemma 2.1. If $w_{1}=u_{1}+i u_{3}$ is a non-constant solution of the boundary problem (1.2), there is no other solution $w_{2}=u_{2}+i u_{4}$ for which the integrals in (2.1) exist and the manifold

lies in $D$.

$$
\begin{aligned}
M_{2}: u_{1} & =u_{1}(x, y), & u_{2}=u_{2}(x, y), \\
u_{3} & =u_{3}(x, y), & u_{4}=u_{4}(x, y), \quad(x, y) \in S,
\end{aligned}
$$

To prove the lemma, assume that a second solution $w_{2}$ exists. On substituting from (2.4) into the integral identity (2.1), the integral over $S$ vanishes. Since $Q$ is positive definite, this implies $Q=0$ and therefore that

$$
p_{1}=p_{2}=p_{3}=p_{4}=0,
$$

hold in $S$. In view of the Cauchy-Riemann equations

$$
\begin{aligned}
& p_{1}=q_{3}, \quad p_{2}=q_{4}, \quad q_{k}=\frac{\partial u_{k}}{\partial y}, \\
& q_{1}=-p_{3}, \quad q_{2}=-p_{4},
\end{aligned}
$$

$w_{1}=$ constant, contrary to hypothesis.

To illustrate the lemma, consider the Levi-Civita problem, for which, in present notation

Let us take

$$
f=e^{-3 v} \sin u \text {. }
$$

One finds

$$
\tau=\frac{\cos u_{2}-\cos u_{1}}{\sin u_{1} \sin u_{2}} e^{3\left(u_{3}+u_{4}\right)}=\frac{\left(\lambda^{-1}-\lambda\right) e^{3\left(u_{3}+u_{4}\right)}}{\cos u_{1}+\cos u_{2}}, \lambda=\frac{\sin u_{2}}{\sin u_{1}} .
$$

where

$$
\begin{aligned}
& a=\frac{\lambda^{2}+\cos ^{2} u_{2}}{1+\cos u_{1} \cos u_{2}} r_{1}^{2}, \quad 2 b=-\left(\lambda r_{1}^{2}+\lambda^{-1} r_{2}^{2}\right), \\
& c=\frac{\lambda^{-2}+\cos ^{2} u_{1}}{1+\cos u_{1} \cos u_{2}} r_{2}^{2}, \quad d=0,
\end{aligned}
$$

$$
u_{1}=\theta_{1}, \quad u_{2}=\theta_{2}, \quad r_{1}^{2}=e^{3 u_{3}}=\left(q_{1} / c\right)^{3}, \quad r_{2}^{2}=e^{3 u_{4}}=\left(q_{2} / c\right)^{3},
$$

from which it is clear that the integrals in (2.1) will exist if $\lambda^{-1}, \lambda$ are regular and $\left|u_{1}\right|+\left|u_{2}\right|<\pi$. The domain $D$ is defined by the inequalities

$$
a>0, \quad \Delta=b^{2}-a c<0 \text {. }
$$

The first is obviously satisfied. In view of

$$
\begin{aligned}
& \Delta=\frac{1}{4} \csc ^{2} u_{1} \csc ^{2} u_{2}\left[\left(r_{1}+r_{2}\right)^{2}-\left(r_{1} \cos u_{2}+r_{2} \cos u_{1}\right)^{2}\right] \\
& \cdot\left(r_{1} \sin ^{2} \frac{u_{2}}{2}-r_{2} \sin ^{2} \frac{u_{1}}{2}\right)\left(r_{1} \cos ^{2} \frac{u_{2}}{2}-r_{2} \cos ^{2} \frac{u_{1}}{2}\right),
\end{aligned}
$$

the second, on returning to the hodograph variables (2.7), amounts to prescribing the inequalities (1.1) pictured by the shaded regions in Figure 1a. 
In section 3 we shall need the reversed Cauchy inequality (6), which in its simplest form states that if

$$
(x, y)=x_{1} y_{1}+x_{2} y_{2}-x_{3} y_{3},
$$

then $(x, x)<0$ implies that

$$
(x, y)^{2} \geqq(x, x)(y, y)
$$

with equality prevailing if and only if the vectors

$$
x=x_{1}, x_{2}, x_{3} ; y=y_{1}, y_{2}, y_{3}
$$

are linearly dependent.

\section{The quadratic form $Q$}

A simple calculation verifies that

$$
\begin{aligned}
a Q & =\left(a p_{1}+b p_{2}-d p_{4}\right)^{2}+\left(a p_{3}+b p_{4}+d p_{2}\right)^{2}-\Delta\left(p_{2}^{2}+p_{4}^{2}\right), \\
\Delta & =b^{2}+d^{2}-a c .
\end{aligned}
$$

Consequently if $a>0$, the quadratic form $Q$ is positive definite if $\Delta<0$, positive semi-definite if $\Delta=0$ and indefinite if $\Delta>0 . \Delta$ is known as the discriminant of $Q$.

The domain $D$ of $E_{4}$ in which $Q$ is positive definite is accordingly defined by the inequalities

$$
a>0, \quad \Delta=b^{2}+d^{2}-a c<0,
$$

the coefficients $a, b, c, d$ being determined by $\tau$ in accordance with (2.3).

The points in $E_{4}$ which correspond to equal functions $w_{1}, w_{2}$ form a two dimensional subspace

$$
S_{2}: u_{2}=u_{1}, u_{4}=u_{3} \text {. }
$$

Likewise the points in $E_{4}$ which correspond to functions $w_{1}, w_{2}$ for which

$$
0<\left|w_{1}-w_{2}\right| \leqq \rho, \quad \rho=\rho\left(u_{1}, u_{3}\right),
$$

form a "neighbourhood"

of $S_{2}$.

$$
D_{\rho}: 0<\left(u_{1}-u_{2}\right)^{2}+\left(u_{4}-u_{3}\right)^{2} \leqq \rho^{2},
$$

If $D_{\rho} \subset D$ and $w_{1}$ is a non-constant solution of (1.2), there is no other solution $w_{2}$ for which the integrals in (2.1) exist and the manifold $M_{2}$ in Lemma 2.1 lies in $D_{\rho}$ or even in $\bar{D}_{\rho}$, i.e., in

$$
\bar{D}_{\rho}: 0 \leqq\left|w_{1}-w_{2}\right| \leqq \rho, \quad \rho=\rho\left(u_{1}, u_{3}\right),
$$

since $w_{1}=w_{2}$ can occur for at most finitely many points of $S$, and the partial derivatives $p_{k}$ in (2.6) will continue to vanish at these points by continuity. Thus we see that if a function $\tau$ can be found so that $D_{\rho} \subset D$, i.e., $Q$ is positive definite in $D_{\rho}$, a non-constant solution $w_{1}$ is locally $\rho$-unique, at least among solutions $w_{2}$ for which the integrals in (2.1) exist. 
We now take up the problem of determining the function $\tau$, so that $Q$ will be positive definite in $D_{\rho}$ for some $\rho=\rho\left(u_{1}, u_{3}\right)$, i.e., so that the inequalities (3.2) hold in $D_{\rho}$.

Let us denote evaluation in $S_{2}$ by a bar, for example

where

$$
\bar{f}_{2}=f_{1}, \quad \bar{f}_{2}^{\prime}=f_{1}^{\prime}, \quad \overline{f_{2}}=f_{1}^{\prime},
$$

$$
f_{1}^{\prime}=\frac{\partial f_{1}}{\partial u_{1}}, \quad f_{2}^{\prime}=\frac{\partial f_{2}}{\partial u_{2}}, \quad f_{1}^{\prime}=\frac{\partial f_{1}}{\partial u_{3}}, \quad f_{2}=\frac{\partial f_{2}}{\partial u_{4}} .
$$

As our first condition on $\tau$, from (2.3) we are led to require

$$
\bar{a}=f_{1} \bar{\tau}_{u_{1}}>0 .
$$

On substituting from (2.3), one finds that

$$
\Delta=U^{2}+V^{2}+W
$$

where

Clearly

$$
\begin{aligned}
U & =\frac{1}{2}\left[f_{1} \tau_{u_{1}}+f_{2} \tau_{u_{2}}-\left(f_{1}^{\prime}-f_{2}^{\prime}\right) \tau\right], \\
V & =\frac{1}{2}\left[f_{1} \tau_{u_{3}}+f_{2} \tau_{u_{4}}+\left(\dot{f}_{1}+\dot{f}_{2}\right) \tau\right], \\
W & =f_{1}\left(f_{1}^{\prime}-f_{2}^{\prime}\right) \tau \tau_{u_{1}} .
\end{aligned}
$$

$$
\bar{W}=0, \quad \bar{\Delta}=\bar{U}^{2}+\bar{V}^{2} \geqq 0
$$

and we can secure $\bar{\Delta}=0$, by choosing $\tau$ subject to the conditions

$$
\bar{\tau}_{u_{1}}+\bar{\tau}_{u_{2}}=0, \quad f_{1}\left[\bar{\tau}_{u_{3}}+\bar{\tau}_{u_{4}}\right]+2 \hat{f}_{1} \bar{\tau}=0
$$

where by $\bar{\tau}_{u_{1}}$, for example, we mean the value of $\bar{\tau}_{u_{1}}$ on $S_{2}$ and not the partial derivative of $\bar{\tau}$ with respect to $u_{1}$ which equals $\bar{\tau}_{u_{1}}+\bar{\tau}_{u_{2}}$. We shall prove that $\bar{a}>0, \Delta<0$ in $D_{\rho}$ imply $\bar{\tau}=0$, provided $f_{1}^{\prime \prime} \neq 0$. Indeed $\Delta$ is positive whenever $W$ is positive and $W$ can be written in the form

$$
W=\frac{a}{\lambda}\left(f_{1}^{\prime}-f_{2}^{\prime}\right) \tau, \quad \lambda=f_{2} / f_{1} .
$$

If $\bar{\tau} \neq 0$, the quantities $a, \lambda, \tau$ maintain fixed signs in the neighbourhood of $S_{2}$, but $f_{1}^{\prime}-f_{2}^{\prime}$ will change sign, in as much as $f_{1}^{\prime \prime} \neq 0$. Thus $W$, and $\Delta$ also, would become positive in a neighbourhood of $S_{2}$, contrary to the requirement that $\Delta$ be negative in $D_{\rho}$. Consequently we lay down the following " initial conditions"

$$
\bar{\tau}=0, \quad \bar{\tau}_{u_{1}}+\bar{\tau}_{u_{2}}=0, \quad \bar{\tau}_{u_{3}}+\bar{\tau}_{u_{4}}=0,
$$

for $\tau$, the latter two being obvious consequences of the first.

For $u_{1}, u_{3}$ fixed $\Delta$ becomes a function of $u_{2}, u_{4}$. We seek a function $\tau$ so that $\Delta$ will have zero for a relative maximum at a point $P_{0}\left(u_{1}, u_{3}\right)$ of the $\left(u_{2}, u_{4}\right)$ plane. If this can be done for each point $P_{0}$, we shall have $\Delta<0$ in a domain $D_{\rho}$ as desired. Sufficient conditions for zero to be a relative maximum for $\Delta$ 
at $P_{0}$ are of course

$$
\bar{\Delta}=\bar{\Delta}_{u_{2}}=\bar{\Delta}_{u_{4}}=0, \quad \bar{\Delta}_{u_{2} u_{2}}<0, \quad \bar{\Delta}_{u_{2} u_{4}}^{2}-\bar{\Delta}_{u_{2} u_{2}} \bar{\Delta}_{u_{4} u_{4}}<0 .
$$

From the definition of $\Delta$ one finds

$$
\begin{gathered}
\bar{\Delta}_{u_{2}}=\bar{W}_{u_{2}}=-f_{1} f_{1}^{\prime \prime} \bar{\tau} \bar{\tau}_{u_{1}}=0, \quad \bar{\Delta}_{u_{4}}=\bar{W}_{u_{4}}=-f_{1} f_{1}^{\prime} \bar{\tau} \bar{\tau}_{u_{1}}=0, \\
\bar{\Delta}_{u_{2} u_{2}}=2\left(\bar{U}_{u_{2}}^{2}+\bar{V}_{u_{2}}^{2}\right)+\bar{W}_{u_{2} u_{2}}, \quad \bar{\Delta}_{u_{2} u_{4}}=2\left(\bar{U}_{u_{2}} \bar{U}_{u_{4}}+\bar{V}_{u_{2}} \bar{V}_{u_{4}}\right)+\bar{W}_{u_{2} u_{4}}, \\
\bar{\Delta}_{u_{4} u_{4}}=2\left(\bar{U}_{u_{4}}^{2}+\bar{V}_{u_{4}}^{2}\right)+\bar{W}_{u_{4} u_{4}},
\end{gathered}
$$

in which

$$
\begin{aligned}
& \bar{W}_{u_{2} u_{2}}=2 f_{1} f_{1}^{\prime \prime} \bar{\tau}_{u_{2}}^{2}, \quad \bar{W}_{u_{2} u_{4}}=f_{1} \bar{\tau}_{u_{2}}\left(\dot{f}_{1}^{\prime} \bar{\tau}_{u_{2}}+f_{1}^{\prime \prime} \bar{\tau}_{u_{4}}\right), \\
& \bar{W}_{u_{4} u_{4}}=2 f_{1} \dot{f}_{1}^{\prime} \bar{\tau}_{u_{2}} \bar{\tau}_{u_{4}} .
\end{aligned}
$$

Clearly $\bar{\Delta}_{u_{2} u_{2}}<0$ implies $\bar{W}_{u_{2} u_{2}}<0$, and therefore

One also observes that

$$
f_{1} f_{1}^{\prime \prime}<0, \quad \bar{\tau}_{u_{2}} \neq 0
$$

$$
{\overline{W_{u_{2} u_{4}}^{2}}}^{2}-\bar{W}_{u_{2} u_{2}} \bar{W}_{u_{4} u_{4}}=f_{1}^{2} \bar{\tau}_{u_{2}}^{2}\left(\dot{f}_{1}^{\prime} \bar{\tau}_{u_{2}}-f_{1}^{\prime \prime} \bar{\tau}_{u_{4}}\right)^{2} \geqq 0 .
$$

If inequality holds, $P_{0}$ is a saddle point of $W$, so that $W$, and $\Delta$ also, would be positive in a neighbourhood of $P_{0}$. Therefore equality holds, and we have the additional " initial condition"

$$
f_{1}^{\prime} \bar{\tau}_{u_{2}}-f_{1}^{\prime \prime} \bar{\tau}_{u_{4}}=0
$$

on $\tau$. Assuming that this condition is fulfilled, we write

so that

$$
\bar{W}_{u_{2} u_{2}}=-2 R^{2}, \quad \bar{W}_{u_{2} u_{4}}=-2 R S, \quad \bar{W}_{u_{4} u_{4}}=-2 S^{2}
$$

$$
\begin{aligned}
& \bar{\Delta}_{u_{2} u_{2}}=2\left(\bar{U}_{u_{2}}^{2}+\bar{V}_{u_{2}}^{2}-R^{2}\right), \quad \bar{\Delta}_{u_{2} u_{4}}=2\left(\bar{U}_{u_{2}} \bar{U}_{u_{4}}+\bar{V}_{u_{2}} \bar{V}_{u_{4}}-R S\right), \\
& \bar{\Delta}_{u_{4} u_{4}}=2\left(\bar{U}_{u_{4}}^{2}+\bar{V}_{u_{4}}^{2}-S^{2}\right) .
\end{aligned}
$$

In view of the reversed Cauchy inequality (2.8)

$$
\bar{\Delta}_{u_{2} u_{4}}^{2}-\bar{\Delta}_{u_{2} u_{2}} \bar{\Delta}_{u_{4} u_{4}} \geqq 0 \text {, }
$$

with equality prevailing if and only if

$$
\frac{\bar{U}_{u_{2}}}{\bar{U}_{u_{4}}}=\frac{\bar{V}_{u_{2}}}{\bar{V}_{u_{4}}}=\frac{R}{S} .
$$

Thus the inequalities in (3.6) cannot be fulfilled simultaneously. In place of the last inequality, we are forced to consider the ambiguous case

$$
\bar{\Delta}_{u_{2} u_{4}}^{2}-\bar{\Delta}_{u_{2} u_{2}} \bar{\Delta}_{u_{4} u_{4}}=0
$$

at the critical point $P_{0}$ in our attempt to insure that $\Delta$ has a relative maximum at $P_{0}$.

One readily verifies that

$$
\frac{R}{S}=\frac{\bar{W}_{u_{2} u_{2}}}{\bar{W}_{u_{2} u_{4}}}=\frac{f_{1}^{\prime \prime}}{f_{1}^{\prime}} .
$$


If we introduce

under the assumption that

$$
\omega=-\frac{f_{1}^{\prime \prime}}{f_{1}^{\prime}},
$$

$$
f_{1}^{\prime \prime} \neq 0, \quad f_{1}^{\prime} \neq \equiv 0
$$

i.e., $f_{1}$ is neither a linear function of $u_{1}$, nor an additively separable function of $u_{1}, u_{3}$, equations (3.7), (3.8) become

$$
\bar{\tau}_{u_{2}}+\omega \bar{\tau}_{u_{4}}=0, \quad \bar{U}_{u_{2}}+\omega \bar{U}_{u_{4}}=0, \quad \bar{V}_{u_{2}}+\omega \bar{V}_{u_{4}}=0
$$

From the first of these equations we see that

$$
\begin{aligned}
& \bar{\tau}_{u_{2} u_{1}}+\bar{\tau}_{u_{2} u_{2}}+\omega\left(\bar{\tau}_{u_{4} u_{1}}+\bar{\tau}_{u_{4} u_{2}}\right)+\omega^{\prime} \bar{\tau}_{u_{4}}=0, \\
& \bar{\tau}_{u_{2} u_{3}}+\bar{\tau}_{u_{2} u_{4}}+\omega\left(\bar{\tau}_{u_{4} u_{3}}+\bar{\tau}_{u_{4} u_{4}}\right)+\dot{\omega} \bar{\tau}_{u_{4}}=0,
\end{aligned}
$$

and when these equations are used to eliminate the partial derivatives of the first two orders of $\tau$ on $S_{2}$ from the last two equations, we obtain an overdetermined system

$$
f_{1} \omega^{\prime}+f_{1}^{\prime} \omega+f_{1}^{\prime} \omega^{2}=0, \quad f_{1} \dot{\omega}-f_{1}^{\prime}-f_{1} \omega=0,
$$

of partial differential equations for $f_{1}$. From the lemma in the appendix it follows that the function $f_{1}$ must be one of the following types

$$
\text { (i) } f_{1}=g\left(m u_{1}+n u_{3}\right) \text {, (ii) } f_{1}=\bar{f}_{1}+g\left(\frac{u_{3}-\bar{u}_{3}}{u_{1}-\bar{u}_{1}}\right) \text {, }
$$

where $g=g(\xi)$ is an arbitrary function and $m, n, \bar{u}_{1}, \bar{u}_{3}, f_{1}$ denote arbitrary constants.

Summing up our results, we see that the conditions (3.6) for $\Delta$ to have zero for a relative maximum at $P_{0}$ cannot be satisfied simultaneously. If the first four are satisfied, the ambiguous case (3.9) arises at the critical point $P_{0}$ and if $f_{1}$ is neither a linear function of $u_{1}$, nor an additively separable function of $u_{1}, u_{3}$, it must be one of the types (3.10). Boundary problems of the latter two types have been studied by Cushing (5) in his thesis and Dunninger (7) has obtained uniqueness theorems if $f_{1}$ is of type (i) in (3.10) in the special cases

$$
f_{1}=e^{m u_{1}+n u_{3}}, \quad f_{1}=\left(m u_{1}+n u_{3}\right)^{1+p}, \quad m \neq 0, \quad(p=1,2, \ldots) .
$$

4. The case $f=f(m u+n v)$

This section will be devoted to the special case of the boundary problem (1.2)

$$
u_{n}=h(s) f(\xi), \quad \xi=m u+n v, \quad m, n=\text { const., }
$$

to which we have been led by the considerations of the previous section.

Given a solution $w_{1}=u_{1}+i u_{3}$ of (4.1), a one-parameter family of solutions

$$
w_{2}=w_{1}+i(m+i n) c, \quad c=\text { const. (real), }
$$

is generated by it. Under what conditions are these the only solutions of (4.1)? 
The mapping

$$
\xi_{1}=m u_{1}+n u_{3}, \quad \xi_{2}=m u_{2}+n u_{4},
$$

carries the space $E_{4}$ of the variables $u_{1}, u_{2}, u_{3}, u_{4}$ into the plane $E_{2}$ of the variables $\xi_{1}, \xi_{2}$. This mapping carries the hyperplane

$$
S_{3}: m\left(u_{1}-u_{2}\right)+n\left(u_{3}-u_{4}\right)=0,
$$

in $E_{4}$ into the straight line

$$
S_{1}: \xi_{1}=\xi_{2}
$$

of $E_{2}$. In addition to containing the subspace $S_{2}$ of equal solutions, $S_{3}$ also contains the one-parameter family of solutions (4.2) generated by a given solution $w_{1}$. If $w_{1}, w_{2}$ are two solutions of (4.1), not related by (4.2), the mapping (4.3) carries the region $S$ of the $z$-plane into a set $\Sigma$ of the plane $E_{2}$. Clearly

$$
\left|\xi_{1}-\xi_{2}\right| \leqq\left|w_{1}-w_{2}\right| \sqrt{ }\left(m^{2}+n^{2}\right) \text {. }
$$

Consequently if $w_{1}, w_{2}$ satisfy (3.3') the set $\Sigma$ will be confined to the band

$$
0 \leqq\left|\xi_{1}-\xi_{2}\right|<\rho \sqrt{ }\left(m^{2}+n^{2}\right)
$$

about the straight line $S_{1}$, the level lines $\xi=0$ of the harmonic function $\xi=\xi_{1}-\xi_{2}$, mapping into segments of $S_{1}$.

If we assume that

$$
\tau=\tau\left(\xi_{1}, \xi_{2}\right), \quad \xi_{1}=m u_{1}+n u_{3}, \quad \xi_{2}=m u_{2}+n u_{4},
$$

$U, V, W$ and $\Delta$ become functions of $\xi_{1}, \xi_{2}$. For $\xi_{1}$ fixed $\Delta$ becomes a function of $\xi_{2}$, and in place of (3.6) we now seek a function $\tau$ for which

$$
\bar{\Delta}=\bar{\Delta}_{\xi_{2}}=0, \quad \bar{\Delta}_{\xi_{2} \xi_{2}}<0,
$$

the ${ }^{-}$denoting evaluation on $S_{1}$.

From the definition of $U, V, W$ in (3.5) we now have

where

$$
\begin{gathered}
U=\frac{1}{2} m\left[f_{1} \tau_{\xi_{1}}+f_{2} \tau_{\xi_{2}}-\left(f_{1}^{\prime}-f_{2}^{\prime}\right) \tau\right], \\
V=\frac{1}{2} n\left[f_{1} \tau_{\xi_{1}}+f_{2} \tau_{\xi_{2}}+\left(f_{1}^{\prime}+f_{2}^{\prime}\right) \tau\right], \\
W=m^{2} f_{1}\left(f_{1}^{\prime}-f_{2}^{\prime}\right) \tau \tau_{\xi_{1}},
\end{gathered}
$$

$$
f_{1}=f\left(\xi_{1}\right), \quad f_{2}=f\left(\xi_{2}\right), \quad f_{1}^{\prime}=f^{\prime}\left(\xi_{1}\right), \quad f_{2}^{\prime}=f^{\prime}\left(\xi_{2}\right) .
$$

One readily verifies that, in as much as $\bar{\Delta}=\bar{W}=0$,

$$
\bar{U}=\frac{1}{2} m f_{1}\left(\bar{\tau}_{\xi_{1}}+\bar{\tau}_{\xi_{2}}\right)=0, \quad \bar{V}=\frac{1}{2} n\left[f_{1}\left(\bar{\tau}_{\xi_{1}}+\bar{\tau}_{\xi_{2}}\right)+2 f_{1}^{\prime} \bar{\tau}\right]=0,
$$

and therefore that

$$
\bar{\tau}=\bar{\tau}_{\xi_{1}}+\bar{\tau}_{\xi_{2}}=0
$$

in consequence of which

$$
\bar{\Delta}_{\xi_{2}}=0, \quad \bar{\Delta}_{\xi_{2} \xi_{2}}=2\left[\bar{U}_{\xi_{2}}^{2}+\bar{V}_{\xi_{2}}^{2}\right]+\bar{W}_{\xi_{2} \xi_{2}} .
$$


If we assume that $\tau$ is regular analytic in the neighbourhood of $S_{1}$, in particular permits the expansion

$$
\tau=\alpha_{1}\left(\xi_{2}-\xi_{1}\right)+\frac{1}{2} \alpha_{2}\left(\xi_{2}-\xi_{1}\right)^{2}+\ldots, \quad \alpha_{k}=\alpha_{k}\left(\xi_{1}\right),
$$

one has

$$
\bar{\tau}=0, \quad \bar{\tau}_{\xi_{1}}=-\alpha_{1}, \quad \bar{\tau}_{\xi_{2}}=\alpha_{1}, \quad \bar{\tau}_{\xi_{1} \xi_{2}}=\alpha_{1}^{\prime}-\alpha_{2}, \quad \bar{\tau}_{\xi_{2} \xi_{2}}=\alpha_{2},
$$

and the formulas for $\bar{U}_{\xi_{2}}, \bar{V}_{\xi_{2}}, \bar{W}_{\xi_{2} \xi_{2}}$ simplify to

$$
\begin{gathered}
\bar{U}_{\xi_{2}}=\frac{1}{2} m\left(f_{1} \alpha_{1}^{\prime}+f_{1}^{\prime} \alpha_{1}\right), \quad \bar{V}_{\xi_{2}}=\frac{1}{2} n\left(f_{1} \alpha_{1}^{\prime}+3 f_{1}^{\prime} \alpha_{1}\right), \\
\bar{W}_{\xi_{2} \xi_{2}}=2 m^{2} f_{1} f_{1}^{\prime \prime} \alpha_{1}^{2} .
\end{gathered}
$$

When these are inserted in the expression for $\bar{\Delta}_{\xi_{2} \xi_{2}}$ above, one finds

$$
\bar{\Delta}_{\xi_{2} \xi_{2}}=\frac{1}{2}\left(m^{2}+n^{2}\right)\left[\left(f_{1} \alpha_{1}^{\prime}+(1+2 l) f_{1}^{\prime} \alpha_{1}\right)^{2}+4(1-l)\left(f_{1} f_{1}^{\prime \prime}+l f_{1}^{\prime 2}\right) \alpha_{1}^{2}\right]
$$

where

$$
l=n^{2} /\left(m^{2}+n^{2}\right), \quad 0 \leqq l<1, \quad m \neq 0 .
$$

To make $\bar{\Delta}_{\xi_{2} \xi_{2}}$ negative, we set

This implies

$$
f_{1} \alpha_{1}^{\prime}+(1+2 l) f_{1}^{\prime} \alpha_{1}=0
$$

$$
\alpha_{1}=k f_{1}^{-1-2 l}, \quad k=\text { const. }
$$

Under these circumstances $\bar{\Delta}_{\xi_{2} \xi_{2}}$ will be negative if and only if $f_{1}$ satisfies the inequality

$$
f_{1} f_{1}^{\prime \prime}+l f_{1}^{\prime 2}<0 \text {. }
$$

A simple calculation verifies that, if $k=-1 / m$ in (4.6), $\bar{a}=f_{1}^{-2 l}$, so that $\bar{a}$ will be positive as long as $f_{1}$ is positive.

Summing up our results, we have the following theorem.

Theorem 4.1. If the function $f(u, v)$ in the boundary problem (1.2) has the special form

$$
f=f(\xi), \quad \xi=m u+n v, \quad m, n=\text { const. }, \quad m \neq 0,
$$

and $f(\xi)$ fulfils the inequality

$$
f f^{\prime \prime}+l f^{\prime 2}<0, \quad l=n^{2} /\left(m^{2}+n^{2}\right), \quad 0 \leqq l<1,
$$

apart from the solutions (4.2) any non-constant solution $w_{1}=u_{1}+i u_{3}$ of (4.1) for which

$$
f_{1}=f\left(\xi_{1}\right)=f_{1}\left(m u_{1}+n u_{3}\right)>0 \text { in } S+C
$$

is locally $\rho$-unique for some function $\rho=\rho\left(\xi_{1}\right)>0$.

Under the conditions of the theorem $\tau$ may be chosen so that $Q$ is positive semi-definite on $S_{1}$ and positive definite in a neighbourhood

$$
0<\left|\xi_{1}-\xi_{2}\right|<\delta, \quad \delta=\delta\left(\xi_{1}\right),
$$

of $S_{1}$ for some function $\delta\left(\xi_{1}\right)$. If we choose $\rho=\delta / \sqrt{ }\left(m^{2}+n^{2}\right)$ in $\left(3.3^{\prime}\right)$, it 
follows from (4.4) that the set $\Sigma$ into which $S$ is carried by the mapping (4.3) will be confined to the strip

$$
0 \leqq\left|\zeta_{1}-\xi_{2}\right|<\delta \text {. }
$$

If there is a second solution $w_{2}$ of (4.1) for which (4.8) holds, the fundamental identity (2.1) implies $p_{1}=p_{2}=p_{3}=p_{4}=0$, and $w_{1}=$ const., contrary to hypothesis. Consequently such a solution $w_{2}$ cannot exist. This result can be extended to include equality as in (4.9), for, the solutions $w_{2}$ in (4.2) being excluded, equality can occur only on the level lines $\xi_{1}-\xi_{2}=0$ in $S$ and the partial derivatives $p_{k}$ will continue to vanish on these level lines by continuity.

That functions $f(\xi)$ exist for which the inequality (4.7) holds can be seen by simple examples. If $f=\xi^{1 / p}$, one readily verifies that

$$
f f^{\prime \prime}+l f^{\prime 2}=\frac{1+l-p}{p^{2} \xi^{2(1-q)}}, \quad q=1 / p
$$

and consequently (4.7) holds for $\xi>0$ provided $p>1+l$. More generally if

we have

$$
f=g^{1 / p}, \quad g=g(\xi),
$$

$$
f f^{\prime \prime}+l f^{\prime 2}=\frac{(1+l-p) g^{\prime 2}+p g g^{\prime \prime}}{p^{2} g^{2(1-q)}}, \quad q=1 / p .
$$

If $p$ is an odd integer greater than $1+l$ and $g g^{\prime \prime} \leqq 0$, inequality (4.7) holds, provided $g^{\prime}$ does not vanish simultaneously with $g g^{\prime \prime}$, e.g., $g=\sin \xi$.

\section{The case $f=f(u)$}

The restriction $f_{1}>0$ in Theorem 4.1 is an unhappy one. Clearly it is not met in the Levi-Civita problem, in which $\sin \theta=0$ at the crests and troughs of the waves. In this section we show how the restriction can be removed in the special case in which $f$ in (1.2) does not depend on $v$. This question has also been treated with other methods by Cushing (5).

For the function $\tau$ we take

$$
\tau=\alpha\left(u_{1}\right) \phi(\lambda), \quad \lambda=f_{2} / f_{1}, \quad f_{1}=f\left(u_{1}\right), \quad f_{2}=f\left(u_{2}\right),
$$

where $\alpha\left(u_{1}\right), \phi(\lambda)$ are two analytic functions at our disposal. We assume that $\bar{\phi}=\phi(1)=0$, to insure that $\tau$ vanishes on the straight line $S_{1}: u_{1}=u_{2}$. Using (2.3) one verifies that

$$
\begin{aligned}
a & =\alpha^{\prime} f_{2} \phi-\alpha f_{1}^{\prime} \lambda^{2} \phi^{\prime}, \\
2 b & =\left[\alpha\left(f_{2}^{\prime}-f_{1}^{\prime}\right)-\alpha^{\prime} f_{1}\right] \phi+\alpha \lambda\left(f_{1}^{\prime}+f_{2}^{\prime}\right) \phi^{\prime}, \\
c & =-\alpha f_{2}^{\prime} \phi^{\prime}, \quad d=0 .
\end{aligned}
$$

Consequently the integrals in the fundamental identity (2.1) exist, provided the ratio $\lambda$ is regular analytic in $S+C$.

E.M.S. - C 
For $u_{1}$ fixed $\Delta$ becomes a function of $u_{2}$ and in place of (4.5) we seek $\tau$ so that

where, from (3.5)

$$
\bar{\Delta}=\bar{\Delta}_{u_{2}}=0, \quad \bar{\Delta}_{u_{2} u_{2}}<0 .
$$

$$
\begin{gathered}
\Delta=U^{2}+W, \quad U=\frac{1}{2}\left[f_{1} \tau_{u_{1}}+f_{2} \tau_{u_{2}}-\left(f_{1}^{\prime}-f_{2}^{\prime}\right) \tau\right], \\
W=f_{1}\left(f_{1}^{\prime}-f_{2}^{\prime}\right) \tau \tau_{u_{1}} .
\end{gathered}
$$

One readily verifies that $\bar{\tau}=0$ implies the first two equalities in (5.2). In addition one has

so that if one chooses

$$
\begin{aligned}
& \bar{\Delta}_{u_{2} u_{2}}=2 \bar{U}_{u_{2}}^{2}+\bar{W}_{u_{2} u_{2}}, \quad \bar{U}_{u_{2}}=\frac{1}{2} \bar{\phi}^{\prime}\left(\alpha f_{1}^{\prime}\right)^{\prime}, \\
& \bar{W}_{u_{2} u_{2}}=2 \bar{\phi}^{\prime 2} \frac{f_{1}^{\prime 2} f_{1}^{\prime \prime}}{f_{1}} \alpha^{2},
\end{aligned}
$$

$$
\Phi^{\prime}=\phi^{\prime}(1)=-1, \quad \alpha=1 / f_{1}^{\prime}
$$

one obtains

$$
\bar{a}=1, \quad \bar{\Delta}_{u_{2} u_{2}}=2 f_{1}^{\prime \prime} / f_{1} .
$$

The case $f=f(m u+n v)$ considered in the previous section reduces to the case $f=f(u)$ if $m=1$ and $n=0$. Theorem 4.1 still applies, but in addition we have the following theorem.

Theorem 5.1. If the function $f(u, v)$ in the boundary problem (1.2) has the special form

and $f(u)$ fulfils the inequality

$$
f=f(u),
$$

apart from the solutions

$$
f^{\prime \prime} \mid f<0
$$

$$
w_{2}=w_{1}+i c, c=\text { const. (real), }
$$

any non-constant solution $w_{1}=u_{1}+i u_{3}$ for which

$$
f_{1}^{\prime}=f^{\prime}\left(u_{1}\right) \neq 0 \text { in } S+C
$$

is locally $\rho$-unique for some function $\rho=\rho\left(u_{1}\right)>0$ among the analytic functions $w_{2}=u_{2}+i u_{4}$ for which $\lambda=f_{2} / f_{1}$ is regular analytic in $S+C$.

If, for example, $f=\sin u$, the hypotheses of the theorem are met for a non-constant solution $w_{1}=u_{1}+i u_{3}$ provided $0 \leqq\left|u_{1}\right|<\pi / 2$ on $S+C$.

\section{Appendix}

This section contains a proof of the following lemma

Lemma. The only solutions of the over-determined system

$$
z w_{x}+z_{x} w+z_{y} w^{2}=0, \quad z w_{y}-z_{x}-z_{y} w=0, \quad w=-z_{x x} / z_{x y}
$$


are developable surfaces $z=z(x, y)$, and are either those with parallel, horizontal rulings

or are cones

$$
z=g(m x+n y), \quad m, n=\text { const., }
$$

$$
z=z_{0}+\left(x-x_{0}\right) g\left(\frac{y-y_{0}}{x-x_{0}}\right), \quad x_{0}, y_{0}, z_{0}=\text { const. }
$$

From the integrability condition

$$
w_{x y}-w_{y x}=w\left(z_{x x} z_{y y}-z_{x y}^{2}\right) / z z_{x y}=0
$$

we see that any solution $z=z(x, y)$ is a developable surface. Taking a developable surface to be the envelope of a one-parameter family of planes

$$
z=a x+b y+c
$$

where $a, b, c$ are functions of a parameter $t$, the Cartesian equation of the envelope is obtained by eliminating $t$ between this equation and

$$
\dot{a} x+b y+\dot{c}=0
$$

(where the dots denote differentiation with respect to $t$ ), an equation which defines $t=t(x, y)$ implicitly. One readily verifies that

$$
z_{x}=a, \quad z_{y}=b, \quad z_{x x}=\dot{a} t_{x}, \quad z_{x y}=\dot{a} t_{y}=b t_{x}, \quad z_{y y}=b t_{y},
$$

from which it follows that the developable surface will be a solution of the system if and only if the Wronskian of $a, b, c$ vanishes, i.e., the functions $a, b, c$ are linearly dependent.

If $a, b$ are linearly dependent, say $n a=m b$ for constant $m, n$, the envelope is a ruled surface

$$
z=g(m x+n y)
$$

with parallel, horizontal rulings. If $a, b$ are linearly independent, we can write $a x_{0}+b y_{0}+c=0$, with $x_{0}, y_{0}$ constant, and find that the envelope is a cone

$$
z=z_{0}+\left(x-x_{0}\right) g\left(\frac{y-y_{0}}{x-x_{0}}\right) \text {. }
$$

\section{REFERENCES}

(1) T. LEVI-CivirA, Détermination rigoureuse des ondes permanentes d'ampleur finie, Math. Ann. 93 (1925), 264-314.

(2) L. LichTENSTEIN, Nichtlineare Integralgleichungen (Leipzig, 1931), 47-54.

(3) J. J. Stoker, Water Waves (New York, 1957), 513-543.

(4) D. Dunninger and M. H. Martin, On a uniqueness question of LeviCivita, Atti Accad. Naz. Lincei Rend. Cl. Sci. Fis. Mat. Natur. (8) 41 (1966), 452-459.

(5) J. CuSHING, Local uniqueness for harmonic functions under nonlinear boundary conditions, University of Maryland doctoral thesis (1968). 
(6) M. H. MArtin and G. N. Trytten, Inequalities associated with quadratic forms, Atti. Acc. Sc. Torino, Cl. Sci. Fis. Mat. Natur. 101 (1966-67), 285-290.

(7) D. Dunninger, Uniqueness and comparison theorems for harmonic functions under boundary conditions, J. Math. and Phys. 46 (1967), 299-310.

UNIVERSITY OF MARYLAND

College Park, Maryland 